\title{
Multiscale Characterization of Microstructure and Residual Strain Distribution in Additively Manufactured Inconel 625
}

\author{
Kathryn A. Small ${ }^{*}$, Mitra L. Taheri ${ }^{1}$ \\ 1. Materials Science and Engineering Department, Drexel University, Philadelphia, PA, USA. \\ * Corresponding Author: kas572@drexel.edu
}

Additive manufacturing (AM) has the potential to decrease time of fabrication, material waste, and increase speed of design for complex components. There are many difficulties in the development of reliable AM processes for metal parts, an active area of research which includes surface roughness, mechanical strength, repeatability and residual stress in the final components. Direct metal laser sintering (DMLS) is an AM technique in which a laser is used to sinter layers of metal powder together into a desired shape; DMLS is a violent and complex thermal process which results in non-equilibrium solidification and anisotropic microstructures which in turn can cause decreased mechanical and material properties [1]-[3].

Residual stress is a key feature in materials which can result in undesired bulk distortion of components and can affect corrosion or mechanical properties on a local scale [4]. DMLS results in unpredictable distributions of residual stress due to its complicated thermal history and fast solidification rates [5]. Elastic strain on a subgrain and nanometer scale can be measured using electron microscopy techniques including nanobeam precession electron diffraction (PED) in TEM TopSpin and, more recently, crosscorrelation of high resolution EBSD (HR-EBSD) or transmission Kikuchi diffraction (TKD) data [6]. The linking of length scales in elastic strain measurement presents difficulty, especially on the subgrain scale, because there has not been a reliable method to determine distribution of elastic strain in regions of high misorientation, a complication that is reliably present in metals fabricated by the DMLS process [7]. Recently, the HR-EBSD method has been improved to minimize misorientation between reference and experimental patterns, enabling the mapping of subgrain elastic strain in DMLS materials (Fig. 1) [8].

A Nye tensor calculation results in GND density maps on the subgrain level when used with PED in the TEM or EBSD in the SEM [9]. The resulting dislocation density data allows for comparison of subgrain strain distribution found by HR-EBSD or PED to be compared to intragranular GND densities and cell substructures in non-equilibrium microstructures (Fig. 2). Evaluation of microstructural features in conjunction with local strain will further the understanding of residual stress accumulation in thermally and mechanically processed materials and would allow experimental validation of AM simulations in which evolution of microstructure and properties of residual stress is desired to be predicted for more reliable AM components.

In this work we use a dual-beam FIB serial sectioning method and iterative HR-EBSD cross-correlation to observe elastic strain distribution at varying depths in DMLS fabricated IN625. The resulting elastic strain maps at each depth are compared to GND density maps of the same area resulting from the Nye tensor calculation, enabling visualization of the evolution of microstructure through part depth in DMLS processing. Elastic strain distributions from HR-EBSD are then compared to the elastic strain on the nanoscale calculated using TKD cross-correlation and TEM TopSpin to link local strain on the nano- and micro- scales. Comparison of elastic strain distributions to dislocation density is performed on both length 
scales and cell substructures previously found in laser sintered materials are examined for their relationship to elastic strain hotspots [10].

\section{References:}

[1] YSJ Yoo et al., Mater. Sci. Eng. A. 724 (2018), p. 444.

[2] KG Prashanth and J Eckert. J. Alloys Compd. 707 (2017), p. 27.

[3] B Farber et al., Mater. Sci. Eng. A. 712 (2017), p. 539.

[4] JE Scheel, NE Jayaraman and DJ Hornbach. NACE Corrosion Conference and Expo.

(2011), p. 1.

[5] P Mercelis and J-P Kruth, Rapid Prototyp. J. 12 (2006), p. 254.

[6] TB Britton and AJ Wilkinson, Ultramicroscopy 111 (2011), p. 1395.

[7] TB Britton and AJ Wilkinson, Ultramicroscopy 114 (2012), p. 82.

[8] KA Small et al., "Toward Local Residual Stress Measurement in Additive Manufacturing Using High Resolution EBSD," Submitted.

[9] AC Leff, CR Weinberger and ML Taheri, Ultramicroscopy 153 (2015), p. 9.

[10] The authors would like to acknowledge Dr. David Fullwood and Zach Clayburn of Brigham Young Unversity for their help and expertise in HR-EBSD cross-correlation. A CRADA between the authors and U.S. Army ARDEC made this work possible and Matthew Clemente, Elias Jelis and Ryan Carpenter are acknowledged for sample fabrication and process development.
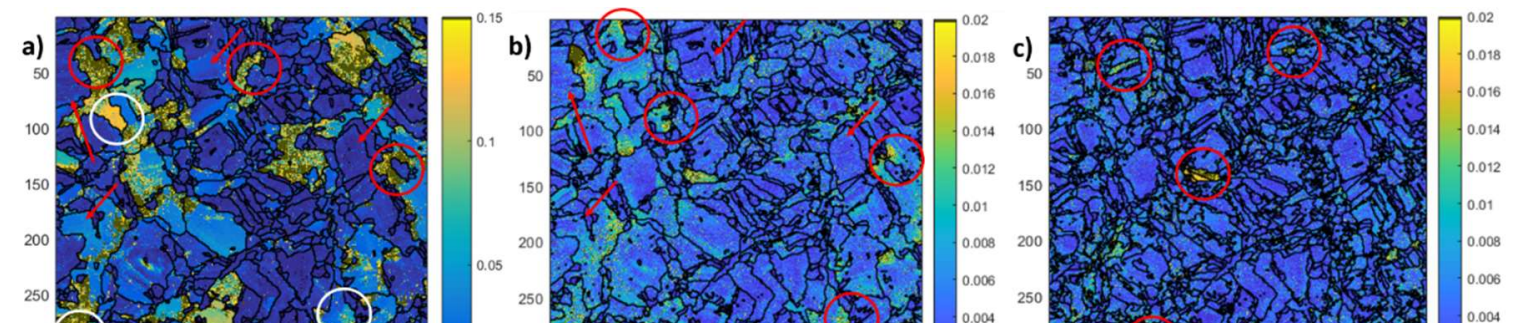

Figure 1. Strain calculation of IN625 fabricated by DMLS. a) Cross-correlation, b) Remapped and c) Subdivided analyses. Circles and arrows show observations of strain vs. microstructural features.

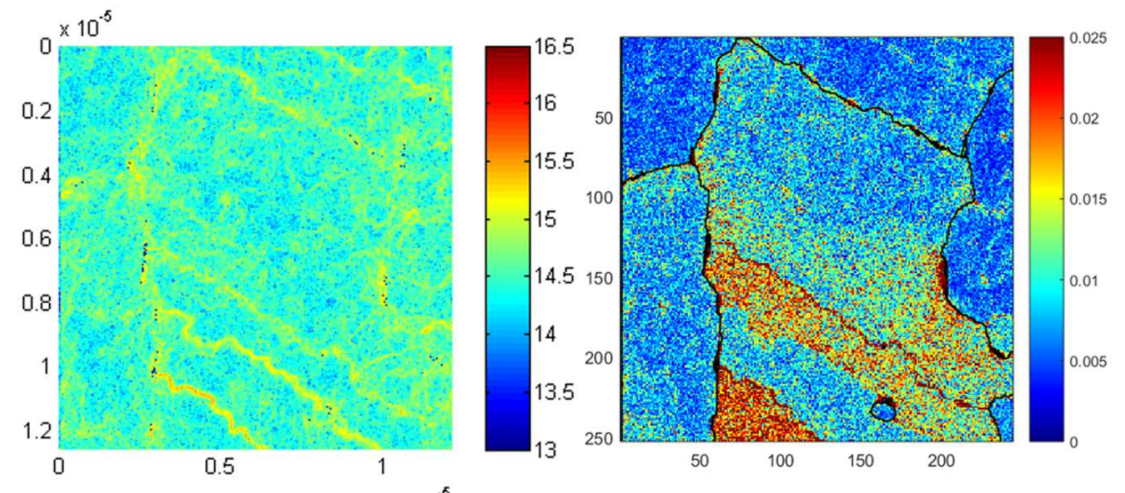

Figure 2. Dislocation density (left) and strain distribution (right) showing cell substructures in DMLS fabricated IN625 